\title{
MODELING HEAT TRANSFER AND SKIN FRICTION FREQUENCY RESPONSES OF A CYLINDER IN CROSS-FLOW - A UNIFYING PERSPECTIVE
}

\author{
Witte A.* and Polifke W. \\ *Author for correspondence \\ Professur für Thermofluiddynamik \\ TU München \\ Boltzmannstr. 15 \\ 85747 Garching \\ Germany \\ E-mail: witte@tfd.mw.tum.de
}

\begin{abstract}
The dynamic behavior of skin friction and heat release of a cylinder in pulsating cross-flow are investigated. Existing analytical solutions are presented as transfer functions versus frequency, known from control theory. Newly found expressions are given for Reynolds number ranges, where no appropriate model exist until now. These expressions are obtained by the combination of CFD simulation and system identification (CFD/SI).
\end{abstract}

In the CFD/SI approach time series are generated by exciting inlet velocity fluctuations over a wide range of frequencies in one single CFD simulation. Time series are acquired for heat release, skin friction and velocity forcing, and then post-processed with system identification tools. Direct numerical simulations are conducted for mean flow Reynolds numbers between 0.1 and 40 , solving the incompressible Navier-Stokes equations in a 2D domain using a finite volume approach. The system identification framework provides methods to identify a mathematical model for the response in heat release and skin friction to velocity fluctuations from data series.

It can be confirmed that Bayly's model for heat release fluctuations performs well at low Reynolds numbers. Lighthill's model, often used in the assessment of Rijke tubes, is more accurate for high Reynolds numbers, but the time constant was underpredicted for Reynolds numbers of order 10. For the range above a Reynolds number of 0.4 a unifying model could be developed. This model especially excels at Reynolds numbers of order 10 . Available models for skin friction usually match the simulated data up to a point, but do not give any dependence on Reynolds number which is corrected here.

The expressions presented allow insight in the physics of the dynamic behavior of a cylinder in pulsating cross flow and also facilitate the use of these models in further investigations.

\section{INTRODUCTION}

The flow around a cylinder in cross-flow is a fundamental problem in thermo-fluiddynamics. Its steady-state behavior has

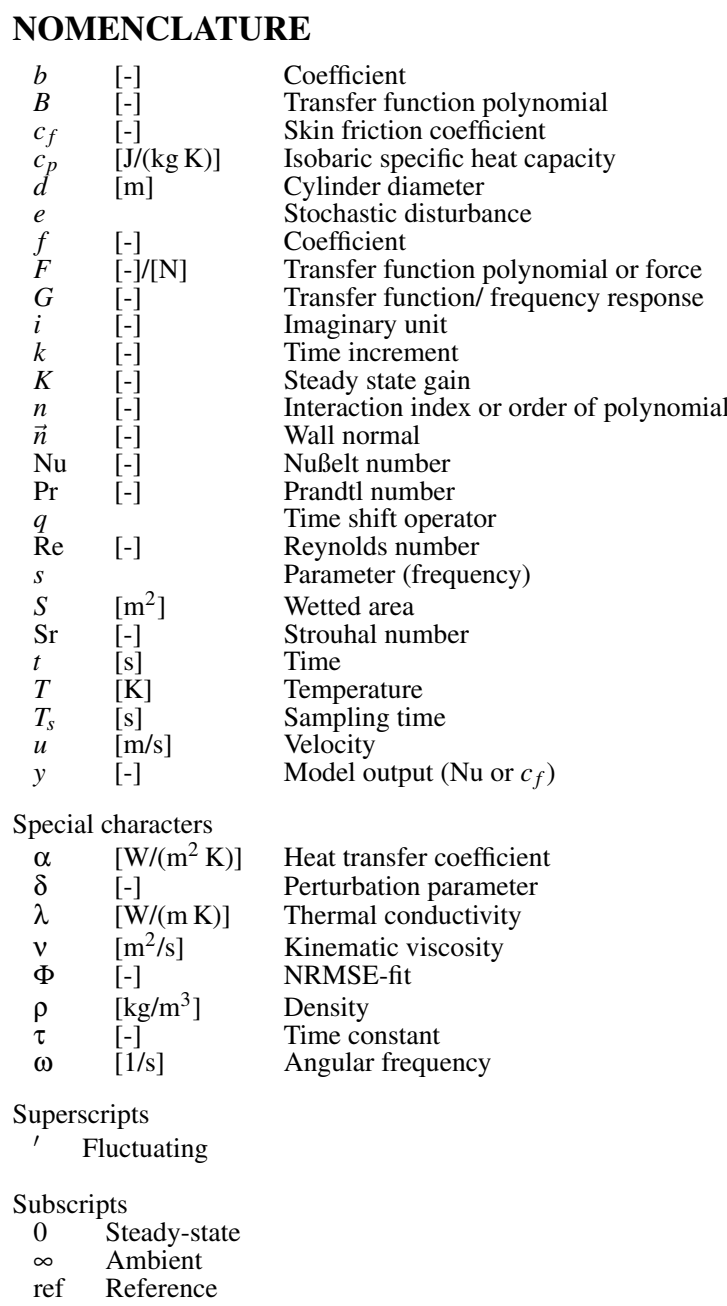

been subject to extensive research and details can be found in many textbooks [1]. The problem of a cylinder in pulsating cross-flow is explored in far less detail. In many applications, however, flow may not be steady, but fluctuating. In a Rijke tube $[2 ; 3 ; 4]$ self-sustained oscillations occur because of the time 
lagged behavior of the heat release of a heated wire in crossflow. The dynamic behavior is also of interest in other applications like hot wire anemometry [5], domestic boilers [6] or gas turbines [7]. Naturally, the dynamics of the velocity field have a great impact on the heat transfer. The flow field is investigated in terms of skin friction which, in addition, is the major part of the drag at low Reynolds numbers. Thus, also the dynamic behavior of the drag on a cylinder is characterized. The impact of pulsating flow on heat transfer and skin friction of a cylinder was investigated analytically and numerically in the past, but the respective models were presented in different frameworks and with different limitations.

The first study was published by Lighthill [8] and is one of the most recognized until now. Under the assumption that the dynamics of a cylinder are mainly governed by the stagnation point flow at the front quarter, the boundary layer equations for this case ("Hiemenz layer") were solved approximately. This led to two solutions for skin friction and heat release; one for low frequencies and one for high frequencies.

Gersten [9] solved the same equations numerically, using a series expansion in frequency. Similar results were achieved, but with more consistent values for intermediate frequencies. Especially the description of the skin friction dynamics is more consistent. Solutions were developed for stagnation point flow and flow over a flat plate ("Blasius layer").

Starting with the Oseen equations, Bayly [10] developed a model for the unsteady heat transfer at very low Péclet numbers i.e. $\operatorname{Re} \operatorname{Pr} \ll 1$ to be used for wires in Rijke tubes or hot wire anemometers. Oseen's approximations allowed for the problem to be solved for a real cylindrical geometry. The skin friction was not addressed in this study.

This work presents the existing analytical solutions in a unifying framework, namely as transfer functions versus frequency. This representation is well known from control theory and allows the use of ubiquitous dimensionless numbers like Reynolds and Prandtl numbers for the problem description. Expressions for a range of Reynolds numbers, where no appropriate model exist until now, are given. These expressions are achieved by the combination of CFD simulation and system identification (CFD/SI).

\section{PROBLEM DESCRIPTION}

In the problem at hand a cylinder is subject to a laminar crossflow with temperature difference $\Delta T$ between cylinder surface and ambient conditions. The velocity $u$ of the approaching flow is fluctuating in time, which leads to fluctuations in heat release and skin friction. Figure 1 depicts a sketch of the problem.

The flow around a cylinder is described by the Navier-Stokes equations. Some assumptions are made a priori to simplify the equations and to facilitate comparison with previous studies. Fluctuations are assumed to travel instantaneously i.e. the cylinder is acoustically compact. Furthermore, a constant temperature difference $\Delta T$ between cylinder and free field facilitates the heat transfer and fluid properties, namely density $\rho$, viscosity $v$, heat conductivity $\lambda$ and heat capacity $c_{p}$ are assumed to be constant. Thus, the ambient and cylinder temperatures are not part of this

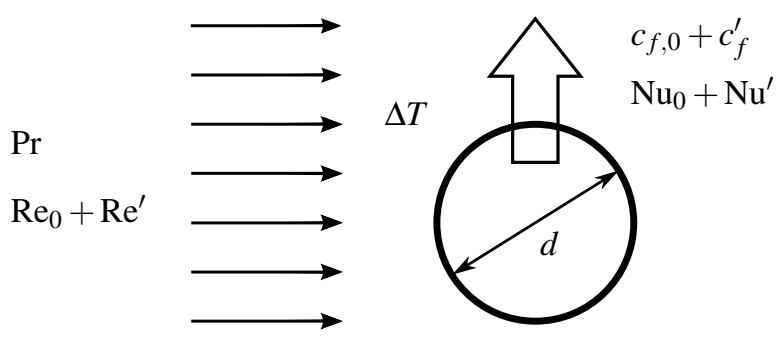

Figure 1. Sketch of the problem: A cylinder (diameter $d$ ) in pulsating cross-flow (Reynolds number Re, Prandtl number Pr) with temperature difference $\Delta T$ between cylinder and far-field causing fluctuating heat release rate (Nußelt number $\mathrm{Nu}$ ) and skin friction (friction coefficient $c_{f}$ ).

problem set. Invoking these assumptions, only the incompressible Navier-Stokes equations and a scalar transport equation for the temperature have to be solved.

Characteristic scales are the cylinder diameter $d$ and the unperturbed free stream velocity $u_{0, \infty}$. The dimensionless Reynolds and Prandtl numbers can be given as

$$
\operatorname{Re} \equiv \frac{u d}{v} \quad \text { and } \quad \operatorname{Pr} \equiv \frac{v \rho c_{p}}{\lambda}
$$

where $\operatorname{Pr}=0.71$ is chosen as a typical value for air. Nußelt number and skin friction coefficient can be written as

$$
\mathrm{Nu} \equiv \frac{\alpha d}{\lambda}=\frac{d}{\Delta T} \frac{\partial T}{\partial \vec{n}} \quad \text { and } \quad c_{f} \equiv \frac{F}{\frac{1}{2} \rho S u_{0, \infty}^{2}}=\frac{v}{\frac{1}{2} u_{0, \infty}^{2}} \frac{\partial u}{\partial \vec{n}}
$$

to quantify heat transfer and friction drag. Here, $\vec{n}$ denotes the wall-normal direction of the cylinder surface scaled with $d$.

The quantities $\mathrm{Re}, \mathrm{Nu}$ and $c_{f}$ can be seen as the linear combination of a steady value (index ${ }_{0}$ ) and a fluctuation $\left({ }^{\prime}\right)$. Transfer functions linking these fluctuations can be defined as

$$
G_{\mathrm{Nu}}(\mathrm{Sr}) \equiv \frac{\mathrm{Nu}^{\prime}(\mathrm{Sr}) / \mathrm{Nu}_{0}}{\operatorname{Re}^{\prime}(\mathrm{Sr}) / \mathrm{Re}_{0}}, \quad G_{c_{f}}(\mathrm{Sr}) \equiv \frac{c_{f}^{\prime}(\mathrm{Sr}) / c_{f, 0}}{\operatorname{Re}^{\prime}(\mathrm{Sr}) / \mathrm{Re}_{0}} .
$$

The Strouhal number $\mathrm{Sr} \equiv(\omega d) / u_{0, \infty}$ is a non-dimensional expression for the frequency of the fluctuation.

\section{DATA GENERATION AND MODEL IDENTIFICATION}

CFD simulations of the problem at hand are used to generate time series data. The inlet velocity is created as the superposition of a fixed mean value and a fluctuation in time. This fluctuation is specifically designed to have high power spectral density over the range of frequencies that are of interest $(0 \leq \mathrm{Sr} \leq 40)$. This excitation never exceeds $30 \%$ of the mean flow, thus the response is linear and time invariant [11].

The desired quantities; heat release, skin friction and inlet velocity are acquired from the simulation for every time increment that is calculated until all relative residuals are below a threshold of $10^{-6}$. This is achieved using a merged PISO/SIMPLE algorithm to solve the incompressible Navier-Stokes equations and a 
transport equation for the temperature by a finite volume method. The algorithms are implemented in the OpenFOAM framework [12] (version 2.3.0).

The computation was performed on a two dimensional grid representing one half of the cylinder, which is justified by the fact that flow below $\operatorname{Re} \approx 47$ is symmetric. Grid spacing and temporal discretization were chosen to resolve the unsteady boundary layer even at high frequencies $(\mathrm{Sr}=40)$, which scales with $(\mathrm{ReSr})^{-1 / 2}$, reasonably well. A total of almost 40,000 cells was used to discretize the computational domain for simulations with $0.1 \leq \operatorname{Re} \leq 4$ and 158,000 for $4<\operatorname{Re} \leq 40$. This results in a resolution of the cylinder surface by 144 and 288 cells respectively.

The acquired time series, which are at least $200 d / u_{0, \infty}$ long, are used for two different purposes. Firstly, the agreement of existing models with simulation data is assessed and secondly, new models are identified especially in cases where the agreement between response of the existing model and CFD simulated response is poor.

To compare model response and simulated response, a normalized root mean square error (NRMSE) is calculated, using the CFD solution as a reference. The NRMSE-fit is given by

$$
\Phi=\frac{\left\|y_{\text {ref }}-\hat{y}\right\|_{2}}{\left\|y_{\text {ref }}-\overline{y_{\text {ref }}}\right\|_{2}}
$$

where the subscript (ref) is either a CFD simulation or frequency response data. A fit of $\Phi=100 \%$ indicates perfect agreement and values below $\Phi=0 \%$ imply a worse match than just a constant mean value.

The CFD/SI method, combining numerical simulation with system identification was proposed by Polifke et al. [13]. It has been applied to various problems in aero-acoustics and thermoacoustics [7].

For the identification, the chosen model structure is an output error (OE) model which, in discrete time $t=k T_{s}\left(T_{s}\right.$ is the sampling time) takes the form

$$
\frac{y^{\prime}[k]}{y_{0}}=\frac{B(q)}{F(q)} \frac{\operatorname{Re}^{\prime}[k]}{\operatorname{Re}_{0}}+e[k]
$$

with the polynomials

$$
\begin{aligned}
& B(q)=b_{0}+b_{1} q^{-1}+b_{2} q^{-2}+\cdots+b_{\left(n_{b}-1\right)} q^{-\left(n_{b}-1\right)} \text { and } \\
& F(q)=1+f_{1} q^{-1}+f_{2} q^{-2}+\cdots+f_{n_{f}} q^{-n_{f}}
\end{aligned}
$$

denoting the transfer function and a noise term $e[k] . N u$ and $c_{f}$ are is substituted for $y$ depending on which transfer function is identified. The backward-shift operator $\left(q^{-n} x[k]=x[k-n]\right)$ is used to relate to past inputs, and $n_{b}$ and $n_{f}$ are the orders of the polynomials i.e. the model orders. The coefficients of the polynomials $b_{0} \ldots b_{n_{b}}$ and $f_{1} \ldots f_{n_{f}}$ are found during identification by nonlinear regression. The transfer function can easily be converted into an expression for the frequency response or from discrete time to continuous time representation. The system identification procedures are implemented in MATLAB [14].

\section{SKIN FRICTION FREQUENCY RESPONSE}

\section{Comparison to existing models}

The models coming closest to a description of the dynamic behavior of the skin friction are given by Lighthill and Gersten. However, no single model exists for the whole range of Strouhal numbers considered, but only approximations for high and low values of $\mathrm{Sr}$ are given. For this reason the approach of splitting the response in two regimes, low and high frequencies is discussed in the following sections.

All identified transfer functions, using $\mathrm{OE}$ model structure, had at least three coefficients in both polynomials $B(q)$ and $F(q)$ to represent the data accurately. The models fit the acquired data reasonably well $(\Phi>97 \%)$. In addition, the identified parameters vary with Reynolds number. Considering the approach by Lighthill and Gersten to use the boundary layer equations, no dependence on Reynolds number was obtained their models.

Figure 2 shows the amplitude of the complex values frequency response and figure 3 depicts the phase between free stream velocity and skin friction. Solid lines denote identified models for $R e=0.4,4$ and 40. The models by Lighthill and Gersten are represented as dashed lines and dash-dotted lines respectively.

The low and high frequency expressions given by Gersten, in terms of series of $\mathrm{Sr}$, agree well at the point of transition from one to the other $(\mathrm{Sr}=3)$. This allows to identify a transfer function from Gersten's frequency domain model. The identification in this case uses the frequency response evaluated at $0 \leq \mathrm{Sr} \leq 40$ and performs linear regression to obtain model parameters. The model is similar to an OE model (equation 5), but in continuous time. The result can be written as

$$
G_{c_{f}}=\frac{17.28 s^{2}+698.1 s+3338}{s^{2}+144.4 s+1751} .
$$

It is translated into a frequency response by applying $s=i \mathrm{Sr}$ which matches the original series by Gersten to $\Phi=98 \%$ NRMSE. Comparing to CFD data the model provides a fit of about $\Phi=85 \%$ for low Reynolds numbers $(\mathrm{Re}=0.1)$ but it drops to $\Phi=55 \%$ at $\operatorname{Re}=40$. Although Gersten's approach relies on the existence of a boundary layer, the fit at lower Reynolds numbers is better. It predicts lower amplitudes than CFD and this discrepancy increases with Reynolds number.

\section{Low frequency response}

At low frequencies i.e. small values of $\mathrm{Sr}$, Lighthill reports an "anticipation time" for the skin friction of $\tau=0.05 d / u_{0, \infty}$ for the Hiemenz layer and $\tau=1.7 d / u_{0, \infty}$ for the Blasius layer. The pressure gradient necessary to accelerate the flow acts faster on the boundary layer than on the free stream. This creates the impression of the skin friction anticipating a change in velocity which can be seen as a phase lead in the frequency response given by

$$
G_{c_{f}}=K \exp (i \operatorname{Sr} \tau)
$$

where $K$ is the steady-state gain. The steady-state gain is reported by Lighthill to have a value of $K=1.5$, due to the fact 


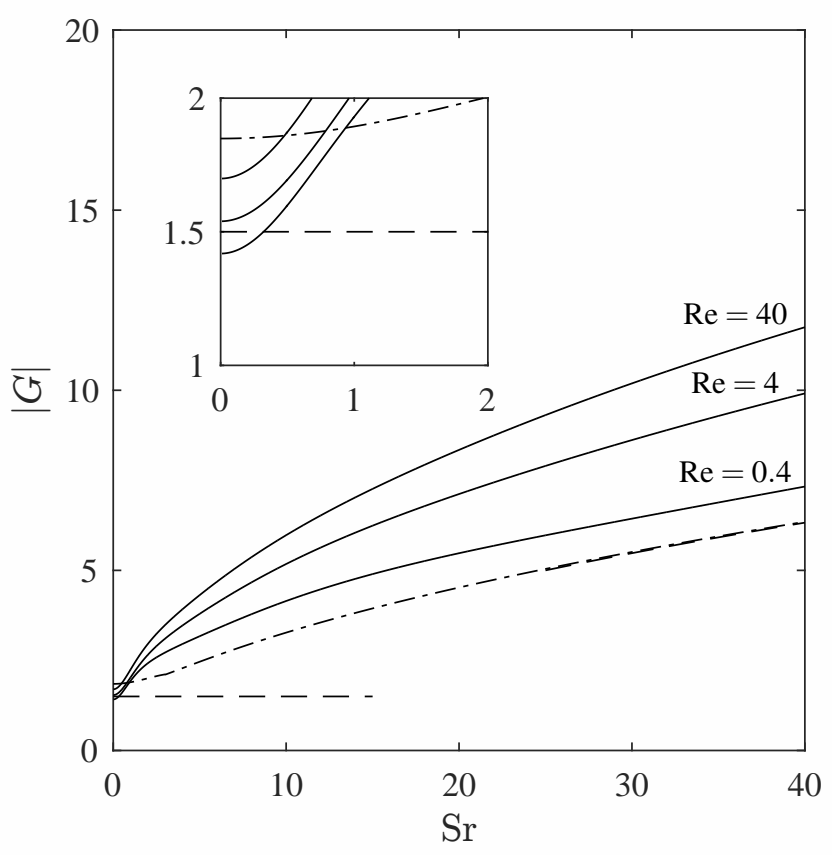

Figure 2. Amplitude of skin friction frequency response to velocity for $\mathrm{Re}=0.4,4$ and 40 (-), Lighthill's approximation $(---)$ and Gersten's results $(-\cdot-)$. The insert magnifies what is the low frequency region.

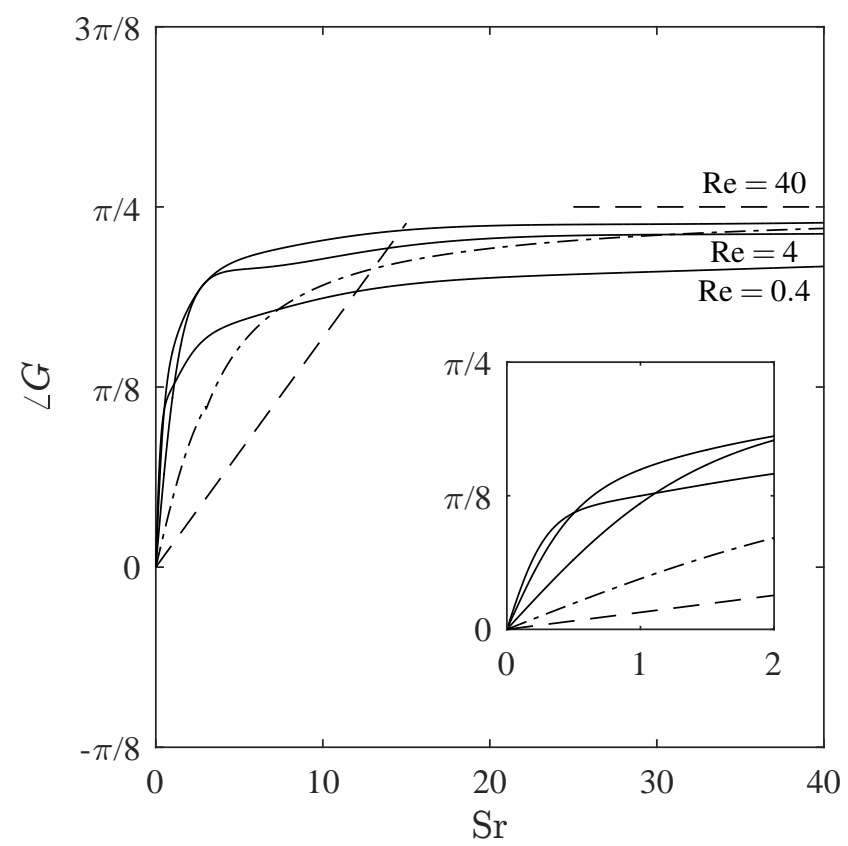

Figure 3. Phase of skin friction frequency response to velocity for $\operatorname{Re}=0.4,4$ and 40 (-), Lighthill's approximation $(---)$ and Gersten's results $(-\cdot-)$. The insert magnifies what is the low frequency region. that $c_{f} \sim u_{0, \infty}^{3 / 2}$. It is stated that the approximation for the phase is applicable for $\mathrm{Sr}<20$.

Gersten's anticipation time is about $\tau=0.15 d / u_{0, \infty}$ and his model shows a smooth transition from low to high frequencies. The applicability of the low frequency approximation is limited to $\mathrm{Sr}<3$ in this case. The low frequency limit is reported to be $K=1.85$.

CFD/SI results show an approximate anticipation time of $0.7 d / u$ which is not depending on Reynolds number. Low frequency limits are changing with Reynolds number with values only slightly above unity for small $\mathrm{Re}$ (regime of linear drag) and approaching $2\left(\operatorname{drag} \sim u_{0, \infty}^{2}\right)$.

\section{High frequency approximation}

At high frequencies all models agree on a square root dependence of the frequency response on Strouhal number. This is closely related to a "shear wave" solution, which also predicts the constant phase lead of $45^{\circ}$. The frequency response may be approximated by

$$
G_{c_{f}}=\left(-1.6 \mathrm{Re}^{-0.1}+3\right) \sqrt{i \mathrm{Sr}}
$$

where the Reynolds number dependence is evaluated by a regression on the data from CFD/SI and is reasonably accurate in the range $0.1 \leq \mathrm{Re} \leq 40$ and $\mathrm{Sr}>5$.

\section{HEAT TRANSFER FREQUENCY RESPONSE Comparison to existing models}

Lighthill presents a model for heat release fluctuations at comparatively high mean flow Reynolds numbers $(\operatorname{Re}>10)$. Bayly gives an expression for the unsteady heat release at low Péclet numbers. Both models are represented in figures 4 and 5 as dashed lines and dash-dotted lines respectively. Solid lines denote models for $R e=0.4,4$ and 40 identified in this work. The figures depict amplitude and phase of the frequency response of heat transfer fluctuations to velocity perturbations.

Time lag and steady-state gain according to Lighthill's approximation for low frequencies are determined to $0.2 \mathrm{~d} / u_{0, \infty}$ and 0.5 respectively. The frequency response thus reads

$$
G_{\mathrm{Nu}}=\frac{0.5}{1+i \operatorname{Sr} 0.2}
$$

Lighthill states that expression (11) is only applicable if $\mathrm{Sr}<20$. However, the heat transfer shows the behavior of a low-pass filter and as the limits

$$
\begin{array}{lll}
\mathrm{Sr} \rightarrow 0: & |G|=0.5 & \angle G=0 \\
\mathrm{Sr} \rightarrow \infty: & |G|=0 & \angle G=-\pi / 2
\end{array}
$$

show; high frequencies have very low gain and a phase lag of $90^{\circ}$, which agrees well with the high frequency approximation.

Assuming a Prandtl number of order unity Bayly gives a limit solution for $\operatorname{Re} \rightarrow 0$. The dimensionless frequency chosen in this work is $\mathrm{Sr} /(\mathrm{Re} \mathrm{Pr})$ and thus includes a dependence on Reynolds number not present in Lighthill's model. Introducing the pertur- 


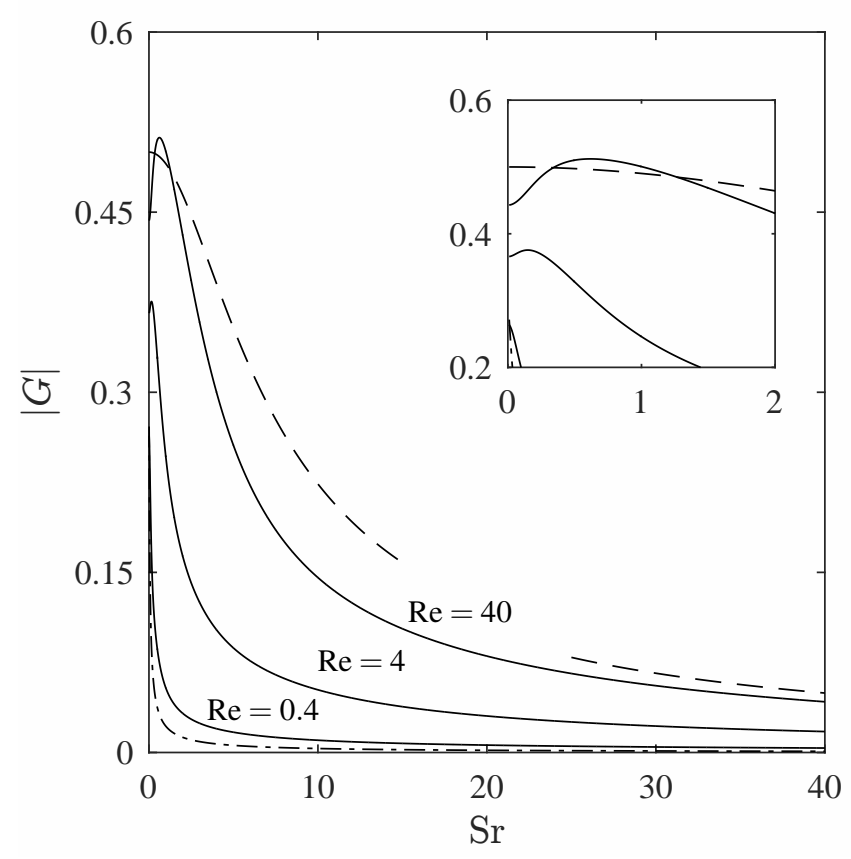

Figure 4. Amplitude of heat release frequency response to velocity for $\mathrm{Re}=0.4,4$ and 40 (-), Lighthill's approximation $(---)$ and Bayly's results $(-\cdot-)$. The insert magnifies what is the low frequency region.

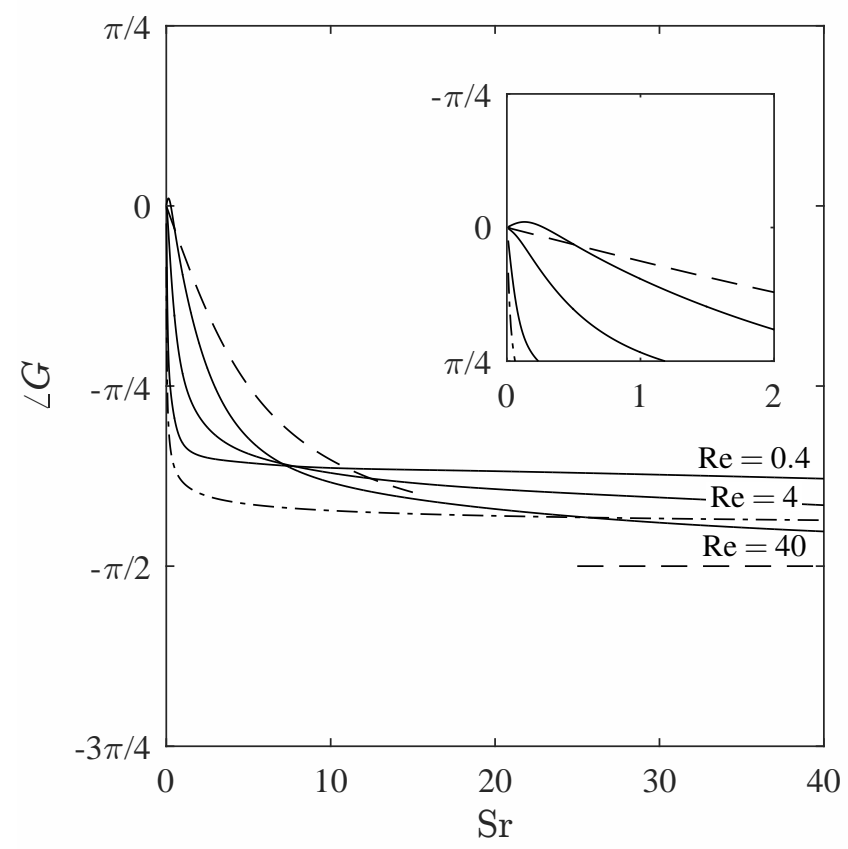

Figure 5. Phase of heat release frequency response to velocity for $\operatorname{Re}=0.4,4$ and 40 (-), Lighthill's approximation $(---)$ and Bayly's results $(-\cdot-)$. The insert magnifies what is the low frequency region. bation parameter $\delta=\left[-\ln (\operatorname{Re} \operatorname{Pr})+\ln (4)-\gamma_{E}\right]^{-1}$ the frequency response can be written as

$$
G_{\mathrm{Nu}}=\frac{\delta \ln (1+4 i \mathrm{Sr} /(\operatorname{Re} \operatorname{Pr}))}{4 i \mathrm{Sr} /(\operatorname{RePr})},
$$

where $\gamma_{E} \approx 0.577$ is the Euler constant. Low and high frequency limits can be denoted by

$$
\begin{array}{lll}
\mathrm{Sr} \rightarrow 0: & |G|=\delta & \angle G=0 \\
\mathrm{Sr} \rightarrow \infty: & |G|=0 & \angle G=-\pi / 2 .
\end{array}
$$

Similar to equation (8) the freqency response given in expression (12) is used to identify a transfer function for the heat release in the form of a ratio of polynomials (equation 5). The analytic frequency response can be approximated by

$$
G_{\mathrm{Nu}}=\delta \frac{1.071 s^{2}+2.909 s+1.173}{s^{3}+5.413 s^{2}+5.247 s+1.173}
$$

as transfer function to very good accuracy $(\Phi=99.88 \%)$. Substituting $s=i \mathrm{Sr} /(\operatorname{Re} \operatorname{Pr})$ yields the frequency response.

Identifying the dynamic behavior of heat release fluctuations from CFD data leads to a range of models depending on Reynolds number. The fit of the identified models to validation time data series is $\Phi>92 \%$ for all Reynolds numbers.

\section{Approximation as first order system}

Following Lighthill's model (equation 11), approximations to the higher order identified transfer functions are given as a first order system. In contrast to the skin friction, this is possible here, because the dynamic behavior is governed to a large extend by only one time lag. Thus, an expression of the form

$$
G_{\mathrm{Nu}}(\mathrm{Sr}, \mathrm{Re})=\frac{K}{1+i \mathrm{Sr} \tau}
$$

is assumed for the frequency response and steady-state gain $K$ and time constant $\tau$ are evaluated to

$$
K=0.25 \operatorname{Re}^{0.2} \quad \text { and } \quad \tau=1.8 \mathrm{Re}^{-0.8}+0.2
$$

by linear regression over 9 identified models in the range $0.4 \leq \operatorname{Re} \leq 40$.

The goodness of fit for the models by Lighthill and Bayly as well as the first order approximation presented here is shown in figure 6. Additionally, the $\Phi$-values for the best identified models are given which correspond to the frequency responses depicted in figures 4 and 5 as solid lines.

As already reported by the respective authors Lighthill's and Bayly's model have limited applicability in the range $0.1<\operatorname{Re}<$ 40. Bayly's model shows high accuracy for $\operatorname{Re} \rightarrow 0$, but should not be used for Reynolds numbers above 0.5. Lighthill states that his model is applicable if a laminar boundary layer exists and gives $\mathrm{Re}>10$ as limit, but better accuracy can be achived by using the Reynolds number dependent model presented here. 


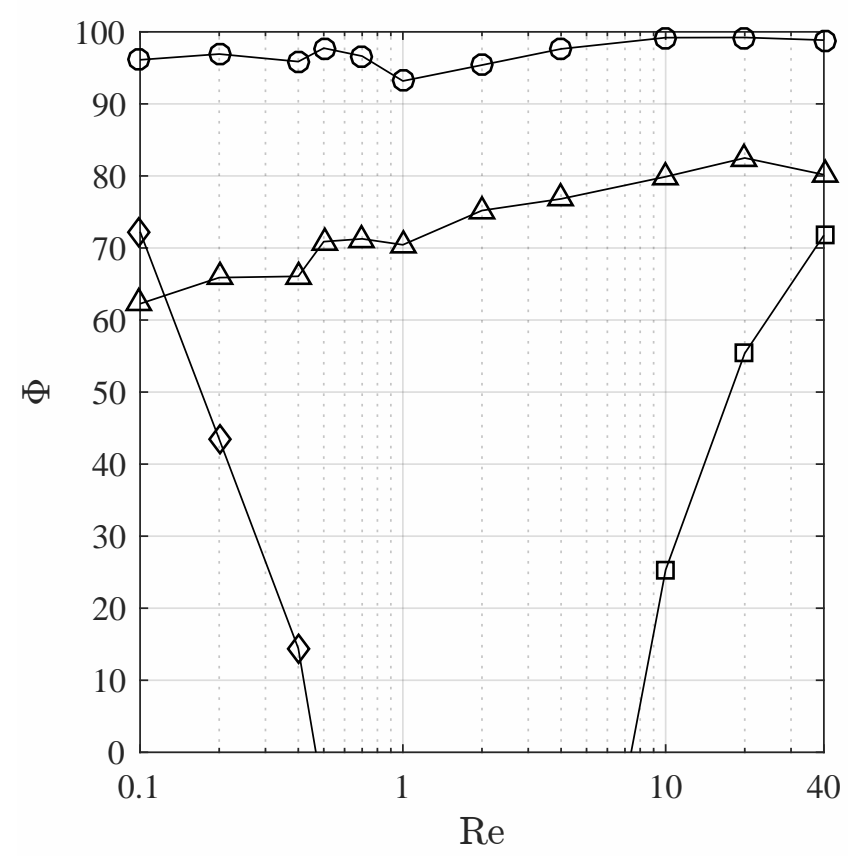

Figure 6. NRMSE-fit of model output from Lighthill ( $\square$ ), Bayly $(\diamond)$, the first order approximation presented here $(\triangle)$ and the identified models providing the best fit (०).

\section{Low frequency approximation}

A model very often used in thermoacoustic applications is that of a time lagged heat release; the so called $n-\tau$ model. It assumes that an output depends only on one past input which occurred some time $\tau$ in the past, scaled with interaction index $n$. Time and frequency domain representations are given by

$$
\begin{aligned}
\frac{\mathrm{Nu}^{\prime}(t)}{\mathrm{Nu}_{0}} & =n \frac{\operatorname{Re}^{\prime}(t-\tau)}{\operatorname{Re}_{0}} \text { and } \\
G_{\mathrm{Nu}} & =n \exp (-i \operatorname{Sr} \tau),
\end{aligned}
$$

similar to the low frequency approximation for $G_{c_{f}}$. It has a constant gain of $n$ and the phase decreases linearly. This behavior only resembles the frequency responses of skin friction at very low frequencies (equation (9)). In this case the Reynolds number dependent constants can be taken from the first order approximation (equation (15)).

The advantage of this approximation is, that the convolution equation, necessary to determine the heat transfer for any velocity perturbation, reduces to a simple multiplication and thus reduces complexity. However, it is important to note that for $\tau \mathrm{Sr}=1$ the difference in amplitude is already more than $49 \%$ and about $12^{\circ}$ in phase. At $\tau \mathrm{Sr}=3$ the amplitude is more than $384 \%$ too high and the difference in phase is greater than $-\pi / 2$.

\section{CONCLUSION}

As can be seen from the results, a complete unification in the manner of a Reynolds analogy is not possible in the dynamic case. However, for the dynamic behavior of the heat release and the skin friction existing models could be translated into transfer functions, which are polynomials in $\mathrm{Sr}$ (or $q$ in discrete time). All analytical models rely on model assumptions that are not valid in the case of Reynolds numbers of order unity or 10. Therefore, new models are identified from simulations using the full incompressible Navier-Stokes equations. These models show a dependence on Reynolds number, which has not been addressed in such detail before. This Reynolds number dependence is used to build new models, which represent an improvement to existing models without introduction too much additional complexity.

\section{ACKNOWLEDGMENT}

Financial support was provided by Deutsche Forschungsgemeinschaft, project PO 710/15-1.

\section{REFERENCES}

[1] T. L. Bergman and Frank P. Incropera, editors. Fundamentals of heat and mass transfer. Wiley, Hoboken, NJ, 7th ed edition, 2011.

[2] Y.-P. Kwon and B.-H. Lee. Stability of the Rijke Tube Thermoacoustic Oscillation. J. Acoust. Soc. Am., 78(78):1414-1420, 1985.

[3] Maria A Heckl. Active Control of the Noise from a Rijke Tube. Journal of Sound and Vibration, 124(Heck188):117-133, 1988.

[4] C C Hantschk and D Vortmeyer. Numerical Simulation of SelfExcited Thermoacoustic Instabilities in a Rijke Tube. J. of Sound and Vibration, 277(3):511-522, 1999.

[5] S. Föller, F. Selimefendigil, and W. Polifke. Linear Identification of the Unsteady Heat Transfer of a Cylinder in Pulsating Crossflow. In Int. Conf. on Jets, Wakes and Separated Flows, Berlin, Germany, September 16-19 2008. Technical University Berlin.

[6] Lin Strobio Chen, Armin Witte, and Wolfgang Polifke. Thermoacoustic characterization of a heat exchanger in cross flow using compressible and weakly compressible numerical simulation. In The 22nd International Congress of Sound and Vibration, Florence, Italy, 12-16 July 2015.

[7] Wolfgang Polifke. Black-box system identification for reduced order model construction. Annals of Nuclear Energy, 67C:109128, May 2014.

[8] M. J. Lighthill. The Response of Laminar Skin Friction and Heat Transfer to Fluctuations in the Stream Velocity. Proceedings of the Royal Society A: Mathematical, Physical and Engineering Sciences, 224(1156):1-23, June 1954.

[9] Klaus Gersten. Heat Transfer in Laminar Boundary Layers with Oscillating Outer Flow. Number 97 in AGARDograph, pages 423-475. 1965.

[10] B. J. Bayly. Heat transfer from a cylinder in a time-dependent cross flow at low Peclet number. Physics of Fluids, 28(12):3451, 1985.

[11] F. Selimefendigil, S. Föller, and W. Polifke. Nonlinear Identification of the Unsteady Heat Transfer of a Cylinder in Pulsating Crossflow. Computers and Fluids, 53:1-14, 2012.

[12] OpenFOAM Foundation. OpenFOAM (Version 2.3.0) [software]. February 2014.

[13] W Polifke, A Poncet, C O Paschereit, and K Döbbeling. Reconstruction of Acoustic Transfer Matrices by Instationary Computational Fluid Dynamics. J. of Sound and Vibration, 245(3):483510, August 2001.

[14] Lennart Ljung. MATLAB and System Identification Toolbox Release 2015b [software]. The MathWorks, Inc., Natick, Massachusetts, United States, February 2014. 